was pronounced by those present to have a distinct]y urinous odour. I myself was unable to express any opinion as a nasal catarrh had taken away my sense of smell. Accordingly the peritoneal cavity was not opened, but a puncture was made in the perinæum and a large catheter passed into the bladder to provide for drainage. The man lived but a few hours afterwards. At the post-mortem examination very acute purulent peritonitis, due to the giving way of a typhoid ulcer, was disclosed. It was not, therefore, a great stretch of the imagination to picture a similar inflammatory exudation as present in this patient, with an escape of some of the fluid along the inguinual canals into the groins, and to a less extent into the scrotum. The comparatively small amount of exudation into the scrotal tissues is probably accounted for by two circumstances(1) the dorsal decubitus of the patient, and (2) the irritating effect of the fluid on the muscular tissue of the scrotum, which was wrinkled as from muscular action of the dartos, and formed a great contrast to the smoothly distended scrotum of the dropsical patient, or that of the sufferer from urinary extravasation. I have mentioned that the cedematous area was excessively tender, to touch it even lightly made the patient start far more than pressure over the abdomen above the level of the extravasation. resembled in this respect the tenderness near the extreme edge of the advancing area in spreading gangrene.

It hardly appears necessary to point out the difference there is between this form of cedema and that due to cellulitis of the scrotum and groins having its origin in some external breach of surface; the limitation of the exudation by the deep layer of the superficial fascia will clearly differentiate the two, as it also excludes any form of dropsy. Its resemblance to extravasation of urine is much more near, but it does not pass into the perineum, and the scrotum in extravasation of urine, where the fluid is at first of a less irritating character, is very much more distended in proportion to the extent of groin extravasation; the penis is also much enlarged, and there is usually a well-marked history of stricture of the urethra, with proof of its presence, whilst there are no symptoms pointing to peritoneal inflammation. If the urethra had given way in front of or under the scrotum the swelling of the penis and scrotum would be in excess of the other portion of the swelling and the stricture signs would be evident, \&c. It is very possible that this condition, although noted, has been ascribed to an exudation through the abdominal wall in consequence of the inflammation of the muscular tissue over a centre of inflammation within the abdomen. It is not unlikely that much of the cedema in cases of localised inflammation about the cæcum has followed the sub-peritoneal tissue in part, and that in an odematous condition of the subcutaneous tissue of the groin the fluid, anticipating the closer approach to the surface of an inflammatory exudation, has really gravitated to that position after its escape from the inguinal canal in consequence of the position of the patient. To say that such a condition as that described does not occur because some observer of known skill has not noted it, recalls an observation to my mind which was made by the President of the Clinical Societiy at a recent meeting; this was to the effect that we could not say that a particular symptom was or was not present in any case, although we might think so, if we had not looked for it. I cannot say that this symptom was not present in the patient to whom I have alluded above, in whom the exudation of fluid into the sub-peritoneal tissue was so excessive, because my attention had not been drawn to the possibility of its occurrence and I dicl not look for it-indeed, the exudation of such an amount of fluid was a surprise to me, but I was informed that a similar exudation of fluid was not uncommonly found in the post-mortem room. If this is the experience of many pathologists, then it is exceedingly probable that the condition to which I have drawn attention is not so uncommon as would at first sight appear to be the case. We have not sufficient data to enable us to form an opinion as to the exact value of this sign, but it is not unlikely that it is one which indicates a more than usually bad prognosis.

Mansfield-street, $\mathbf{W}$.

Wiltshire County CouncrL.-The agricultural committee of the Wiltshire County Council have recommended the appointment of Mr. I. M. H. Munro, D.Sc., F C.S., F.C.I., as district agricultural analyst for the administrative county of Wiltshire.

\section{MEDIASTINAL SURGERY.}

BY H. MILTON, M.R.C.S. ENG.,

PRINCIPAL MEDICAL OFFICEP, KA8R FI AINI HOSPITAL, CAIRO.

THE abdominal and cerebro-spinal cavities and the organs they contain have been freely explored by the surgeon since he has adopted cleanliness with the most brilliant results. The thoracic cavity and its contents, although frequently the seat of surgical interference, have not received the same attention, and no definite lines of technique have so far been laid down. This is partly due to the fact that the thoracic walls present more mechanical difficulties to the surgeon than the abdominal, and partly to the dread with which interference with most of the thoracic organs still inspires him. Having lately met with a series of cases of intra. thoracic disease in which there arose an urgent demand for surgical interference I determined to try to ascertain whether the general cavity of the thorax must of necessity remain a terra incognita to the operating surgeon. Considering the subject in the post-mortem room, the advantages of the median incision-a prolongation, in fact, of the usual abdominal exploratory incision-conld not but strike one. So easy is this incision of execution and so considerable is the power of exploration thereby obtained that one is almost induced to hope that future experience may justify the application to it of the term "normal thoracic incision." $\mathrm{As}$ a commencement of my experiments I opened some tes thoracic cavities of subjects dexd from various diseases, employing, with slight variations, the following method:1. A long skin incision from the thyroid cartilage to the ensiform cartilage. 2. Exposure by rapid dissection. of the trachea down to the notch of the sternum and of the same bone in all its extent. 3 . The separation of the fascia from the notch of the sternum as far outwards as the tendon of the sterno-mastoid on either side, keeping the point of the knife absolutely on the periosteum of the sternum so as to avoid injury to veins, \&c. 4. The separation with the finger tip of the posterior surface of the sternum, just below the notch, from the tissues lying beneath it, these latter being displaced backwards. They consist principally of the innominate vein and artery, the trachea, and many nerve branches and plexuses. 5. An incision with the saw along the middle line of the sternum from notch to ensiform cartilage, not implicating the latter and not quite cutting through the whole thickness of the bone. It is to be noted here that the thickest and hardest portion of the bone is at its upper end. This must be thoroughly divided, the vein and other underlying structures being protected by a finger inserted behind the sternum. 6 . The complete separation of the two halves of the sternum I have carried out in two ways. In some cases I have commenced at the sternal notch, in others just above the ensiform cartilage. The second method has the advantage that the first part of the separation, which has to be done with but a very obscured view of the deeper tissues, takes place at a situation where the mediastinum is wide. There is less danger consequently of wounding the pleura, and by inclining the incision to the left this danger is still more averted. In the operation on the living subject I followed this method, but still I cannow say that I found in my preliminary operations on the cadaver any great superiority of the one over the other method. Whichever method is followed great care is necessary in separatiog the two halves of the sternum to aroid injury of the deeper tissues. In beginning from the lower end of the sternum the ensiform cartilage must be separated first from the sternum. This in young subjects can be done with the knife; in older subjects I have used scisscrs or bone nippers. Strong, broad-hooked retractors are now inserted into the halves of the sternum along the line of incision and moderately firm traction outwards and slightly upwards applied to them. The ensiform cartilage is now thoroughly separated from the sternum. There is but very little possibility of opening the peritoneal cavity in doing this, and even should it occur it is of no importance. With a retractor the tissues lying under the line of separation of the ensiform cartilage and the sternum are now displaced backwards, great care being taken to work quite close up to the posterior surface of this bone. Either with knife or scissors the saw-cut is carried light through the bone, extending upwards to the limit of separation of the deep 
Gissues. Firm retraction is made on either side and a further extent of tissues displaced backwards from the sternum, and the incisions through this latter completed to the same extent. This process is continued with due care until the whole bone is divided. In working from above the process is simple, but perhaps more risky. After thoroughly displacing backwards the deep tissues from behind the sternal motch the upper portion of the bone is completely sawn through so far as the action of the saw can be thoroughly guarded by the finger. The incision along the middle line of the sternum is then completed in the same cautious way as a trephine is usually applied to the cranial bones. A blunt chisel is inserted along this incision, and partly by the twisting of this and partly by the action of the wetractors the two halves of the sternum are forced apart, any necessary assistance being given by careful incisions with the knife. This method I have not carried out on the living, but I believe it to be practicable. 7. Exposure of the mediastina. Firm traction on both halves of the sternum is now sufficient to separate them to the extent of one centimetre, enough to expose any tissues resisting separation to a greater extent. In the cadaver the principal resistance is caused by the ligamentous tissue at the back of the sternal notch. These fibres lie immediately above the innominate vein, but may easily be separated from it and be carefully divided. Another point of resistance is usually at the lower and of the sternum. This may be safely divided, and it will then be found that after dividing any other resisting fibres the two halves of the sternum may be separated to the extent of from five to six centimetres and the anterior mediastinum be thoroughly explored. Crossing the top of this space are seen the innominate vessels, behind them the trachea. The latter can be explored by the finger down to its bifurcation, and can be seen to the same extent if the vessels are thoroughly retracted. Below the vessels are seen the two pleuræ and the pericardium. The latter is a firm structure, and not likely to be injured Auring the exposure of the mediastinum; the former are very delicate in texture, but may with care be left uninjured. In only one of my experiments was the pleura opened, and that was due to carelessness in an emphysematous subject. Once the anterior mediastinum is exposed the exploration of the median and posterior mediastina is a simple matter, and is effected by separation with the fingers of the pericardium from the right pleura. The closure of the mediastinum after exploration is simplicity itself. Six strong silver wires passed first through the skin, then through boles bored at equal distances in both halves of the sternum, and then through the skin again, were sufficient to firmly re-unite the sternum, and in the living subject could be easily removed after cure. A gauze plug situated above the sternal notch and another, if necessary, at the lower end of the sternum would supply sufficient drainage, and a skin suture would complete the closure of the wound.

The results of my experiments on the cadaver sufficed to show the possibility of opening the mediastina through a median incision without injury to any vital structure. I have to thank Dr. Ruffer, the director of the Pathological Department of the Cairo Medical School, for much kindness and assistance. It was now necessary to ascertain what difficulties were to be met with in operating on the living subject. The important questions to be decided were what effect would the opening of the mediastina have on the respiration and circulation; and, further, would the necessary dissection be attended by any considerable or uncontrollable hæmorrhage. The division of the sternum could not fail to seriously affect, if not to arrest, costal respiratory action, but it was to be anticipated that the diaphragmatic action would continue and would suffice. It was, moreover, possible that atmosphere pressure acting on the outside of the pleura might detach it still further from the bony framework of the chest. Precautions were taken, however, to meet these complications should they arise, an apparatus for artificial respiration being prepared with an indiarubber covered glass tube to fit lightly into the trachea. Any considerabie ill exfect on the circulation from opening of the mediastinum was not anticipated, and the result of experiments justified this assumption. The animal chosen was a goat, as being the largest easily obtainable, and the operation was performed in the Physiological Laboratory of the Cairo School of Medicine. (I wish here to express my gratitude to Dr. Wilson, the Professor of Physiology, for great assistance and many useful suggestions.) The animal was chloroformed completely and the thorax shaved. The division of the sternum was a matter of some difficulty, as I did not know its exact shape or relative anatomy. It proved to be very narrow, much more solid than the human sternum, and curved at an acute angle. Moreover, after it had been cut through it became evident that in the goat there is practically no mediastinum, so that the incision in the sternum produced almost of necessity wounds of both pleuræ. An immediate collapse of both lungs was the result. Tracheotomy was performed at once, the glass tube inserted in the trachea and artificial respiration carried out with the bellows. During this process the heart's action became irregular and rapid, but directly respiration was re-established the curculation became normal and remained so during the whole operation. The artificial respiration rapidly produced apnœa, and all respiratory action on the part of the animal ceased, to recommence, however, whenever the artificial respiration stopped. The various mediastina could now be thoroughly explored, and it was found that the loss of blood both during the division of the sternum and the separation of the pleuræ was immaterial. The heart could be thoroughly explored-the pericardial sac was not opened-and it proved very refractory to any rianipulation. A firm grasp on it produced only a temporary irregularity in its action, and immediately on release it resumed its usual rbythm. The condition of the animal remaining very good, I proceeded to close the wound. The pleuræ were closed as far as possible with a continuous silk suture, the sternum re-united with wire sutures, the skin with a continuous silk suture, a gauze plug was placed above the sternal notch, the tube was removed from the trachea as soon as normal respiration had established itself, and a tight dressing was applied round the animal's neck and chest. Half an hour later the goat was walking about the room; it ate and drank, and voided fæces and urine. A short time afterwards it was killed in order to avoid needless suffering.

Having convinced myself that the opening of the mediastina did not of necessity inflict such injury as to entail the death of the individual, I determined to attempt similar operative interference for the relief of human suffering. I have had lately under my care three cases in which the question of surgical interference arose. In the first two the conditions were so entirely against a successful issue that I decided not to make any attempt. The first was a case of mediastinal tumour pressing on the innominate vein, producing cedema of the face, neck, and arm, and on the trachea producing gradually increasing dyspncea. On first seeing the patient it was evident that no hope was left of curative treatment, as there was certainly deposit in the liver and probably in the lungs, and the mediastinal tumour was probably glandular in nature and of secondary malignant growth. I could do nothing but await symptoms of urgent dyspncea and attempt to relieve these by tracheotomy and the insertion of a long tube. The symptoms eventually arose; tracheotomy was attempted, and the patient died on the table. The second patient is still alive, and it was with a view to operative interference in his case that I first commenced my experiments. He was admilted to the hospital suffering from urgent dyspncea of some days' standing, his condition being such that tracheotomy was performed at once by my Egyptian assistant, who was of opinion that the case was one of syphilitic laryngitis. Finding that tracheotomy gave no relief he sent for me. I could find no sign of any mediastinal tumour or other cause of pressure on the trachea, but the obstruction being evidently some distance below the tracheotomy wound I passed a six-inch flexible tracheotomy tube down the trachea, and passed an evident obstruction with immediate relief to the breathing. Careful examination a few days subsequently afforded no definite signs whatever as to the cause of the obstruction. In about fifteen days, however, infiltration began to appear above the sternal notch, and to-day, some two months from the date of tracheotomy, the whole base of the neck is one infiltrated sarcomatous mass. I may say that, in spite of an absence of any syphilitic history, iodide and mercury were thoroughly tried. In the earlier days after tracheotomy the case seemed eminently fitted for mediastinal exploration, but the appearance of infiltration at once set aside all possibility thereof.

My third case was a more favourable one, and after much consideration I operated on the patient on Jan. 25th, 1897, and to-day (Feb, 6th) he may be considered as practically cured. He was an Egyptian fellah aged about twenty-five years. No family history was obtainable. He had suffered for some months (according to his account) from pain and swelling in the sternal region, with increasing incapacity for 
work, and some cardiac palpitation, with pain and oppression in the epigastric region and shortness of breath on exertion. Examination disclosed a very prominent sternal region, the skin over it being infiltrated and tender to pressure. The sternum was the seat of a tumour which occupied nearly its whole length and which extended on either side into the rib area. The tumour was hard in the greater part of its area, softer and tender at others. On percussion the whole tumour area was dull and measured some twenty centimetres in length by seven in breadth. The tumour was elevated about three centimetres above the normal level of the sternum. The heart dulness could not be separated laterally from that of the sternum, but the apex beat was displaced outwards rather more than two and a half centimetres. The heart sounds were normal. The chest expanded well and the breath sounds were normal, except that behind the first part of the sternum the tracheal respiration was harsher than usual. There were no other signs of pressure on the trachea and no signs of pressure on the veins. There was no enlargement of the cervical glands; all the organs of the body were healthy. The pulse was 82 and the temperature was normal in the morning and $37 \cdot 3^{\circ} \mathrm{C}$. at night. Tuberculous caries and necrosis of the sternum being a common disease in Egypt, there being no signs of any sarcomatous growth, and syphilis being absent, a diagnosis was made of infiltrating tuberculosis of the cancellous tissue of the sternum and possibly of the mediastinal glands. The patient being incapacitated from gaining his living and his general condition being good, it was decided to attempt his relief by operation. He was chloroformed and an incision made from the cricoid cartilage to the ensiform cartilage, the skin being reflected on either side and the trachea and sternum exposed. The sternum was soft and friable all over, and larded, as it were, at various points with cheesy, tuberculous deposits. I have on several occasions seen a similar condition in the tibize of tuberculous children; the whole bone is greatly thickened and at the same time porous, and presents at various points deposits the size of a bean of tuberculous, caseating débris. The condition exactly resembles that often found in strumous glands. The sternum was next carefully divided from the ensiform cartilage and was found to be very porous and greatly thickened. This was really the most difficult part of the operation. It would have been easier to have split the ensiform cartilage, but I wished to spare as far as possible the insertion of the diaphragm. The mediastinal tissue underlying the end of the sternum was then depressed and the sternum split with saw and chisel, working little by little intil the bone was divided in its whole length. It was found thickened to the extent of three centimetres in some parts and studded with caseous deposits. In dividing the sternum it was often necessary to remove large portions of infiltrated bone with the gouge. On carefully examining the two halves of the bone they were found to be so infiltrated with tabercle that the whole sternum had to be removed bit by bit with the exception of the upper portion-a centimetre and a half in lengthwhich was simply divided. This division was necessary in order to remove two enlarged caseous glands situated behind it in the mediastinum. After the division of the sternum and separation of its two balves this removal was effected with the greatest ease, the glands peeling as readily off the innominate vein as they would have done off, say, the internal jugular vein. During the whole operation there was remarkably little hamorrhage; half-a-dozen arteries were seized with pressure forceps and a few oozing points were touched with the cautery. At no moment was the heart's action troubled. The costal respiration ceased on the division of the sternum, but the abdominal respiration was maintained with complete regularity. The wound left by the operation was very large-larger, of course, than it need have been in most mediastinal incisions, in that nearly the whole of the sternum had been removed on account of tuberculous deposit. The sides of the cavity were formed by the ends of the costal cartilages and the tissues in between them, at the lower end of the wound was the ensiform cartilage, at the upper end the trachea (exposed in case tracheotiomy became suddenly necessary) and the innominate vessels crossing it. The floor of the cavity was formed by the mediastinal tissue, on either side were the pleure and on the left side the pericardium. The pleura were not adherent to the lungs or the pericardium to the heart, the movements of all three organs being perfectly free. Although aseptic precautions had been taken, I did not dare, considering the tuberculous condition of: the bone, and in spite of the total absence of pus, to attempt complete closure of the wound. I considered it safer to plug with sterile dermatol gauze, which remained unchanged for forty-eight hours. During the whole of this time the patient's condition was most excellent: he had but little shock, his temperature never exceeded $37^{\prime} 1^{\circ} \mathrm{C}$., his respirations were from 24 to 26 and entirely abdominal, his pulse was from 80 to 90 , and he slept. without morphia. On the second day after operation chloroform was again administered and the plug removed. It was found that the ribs had come together to a very considerable. extent, so that the pericardium and pleuræe, though still visible, were but little uncovered. The wound was quite clean, and it was decided to close it. A thin strip of gauze. was laid along the bottom of the wound to serve as a drain, and the soft tissues were closely united from one end of the wound to the other with a continuous wire suture, a small opening being left for the gauze to protrude above the sternal notch and at the lower end of the wound. Two days later the temperature rose to $394^{\circ} \mathrm{C}$., and the respirations to 36 , and there was a slight attempt at coughing. This constituted the only real difficulty in the whole progress of the case. The endeavour to cough was very distressing to the patient, and was practically unavailing. The feeble movements necessary for respiration had been carried on without difficulty, but the violent expulsive action of coughing could not be carried out with a divided sternum. A purgative was given and the dressing changed. On removing the gauze plug $a$. slight retention of secretion, sero-purulent in nature, was discovered. The escape of this secretion was followed by a. fall of temperature to normal, and a cessation of the desire to cough. Three days later there was a rise in temperature to $391^{\circ} \mathrm{C}$. ; a purgation was followed by immediate cessation of these symptoms. To-day the wound is completely dry, the gauze has been removed, and the patient is entirely convalescent. I shall keep him in bed, bowever, for another thirty days at least, in order to allow of thorough consolida. tion. His costal respiration is gradually re-establishing itself, but has not yet returned to the normal. I have to thank Dr. Sandwith, who saw all three cases with me, and who was present at the second operation, for much assistanee and advice.

The practicability of mediastinal exploration has been, I think, established by the above case; experience only can show whether the risk thereby entailed is greater than the profit obtainable. In Cairo we have unfortunately but little literature available, and I cannot be sure whether a similar operation has ever been performed. Péan has, I know, removed a portion of the sternum to get at a mediastinal growth, operating in a diving-bell within which a pressure of two atmospheres was maintained. This information I owe to an assistant at the operation, and I believe that a description is to be found amongst Péan's Cliniques. The operation presented great difficulties and the patient, I believe, died on the second day. It is evident that in an opera tion on the mediastinum there must always be a danger of wounding the pleura; but such a wound, followed as it naturally would be by a collapse of the lung, need not of necessity entail a serious accident. Tuffier and Hallion have shown ${ }^{1}$ that the dog's pleura can be freely opened without ill effect, and I bave myself opened the pleural cavity with impunity on several occasions when operating on liver abscess or tumour. Should any difficulty be anticipated, it is a simple matter to carry out artificial respiration with the bellows through a tracheotomy tube In my experiments I have found that for this a Trendelenburg's cannula is by no means necessary. A bent glass tube covered at its lower end with an indiarubber tube of a smaller diameter and projecting half an inch beyond the glass is quite as effectual and much simpler. Tubes of various diameters should be at hand, and the one inserted which just plugs the trachea. This is quite sufficient to prevent any escape of air at the slight pressure necessary for respiration. If it be established that median thoracic incision is a fairly safe procedure, I have no doubt that it will constitute the most generally useful route to the thoracic organs. And if once a safe route is established a great field for surgical interference lies open. That the lungs are amenable to surgical interference has been frequently proved, and many surgical procedures, from the removal of a foreign body from a bronchus to the extirpation 
of the whole lung, may some time or other be classed amongst established operations. Heart surgery is still quite in its infancy, but it requires no great stretch of fancy to imagine the possibility of plastic operations in some, at all erents, of its valvular lesions. That the heart is tolerant of wounds and injuries has been proved over and over again, and I see no reason why it should not be equally tolerant of a deftly guided scalpel. Surgery of the large thoracic vessels is by no means modern, and an improved technique should render interference with them much easier and much surer. Not only will the anterior mediastinum with its not uncommon growths, innocent and malignant, lie open to us, but also the median and the posterior, and operations on the cesophagus, the thoracic duct, and the great nerve trunks of the pneumogastric and sympathetic may come within our range. Briefly stated, should median thoracic section establish itself as a safe operation the last great cavity of the body and its contents lie open to the surgeon's hand and knife.

Cairo.

\section{REMARKS SUGGESTED BY DR. CLIFFORD ALLBUTT'S PAPER UPON ALBU- MINURIA IN PREGNANCY.}

\section{BY LOVELL DRAGE, M.A., M.D. OXoN.}

To a working obstetrician like myself great pleasure was given by a perusal of this paper, ${ }^{1}$ so full of thought and interest. In a paper upon Puerperal Fevers published in THE LANCET on Feb. 24th and March 3rd, 1894, the following sentence will be found: "The characteristically original and able work of the late Dr. L. G. Wooldridge is suggestive of the idea that the full elucidation of this subject will be derived from chemical investigations, and especially those in connexion with the various conditions of the blood." When I was writing these words I had not the knowledge of such work as I now have, but it was a matter of certainty in my mind that in the case of the puerperal fevers there was a great deal more to be taken into consideration than the simple dictum that puerperal fevers were the result of dirt and nothing else. This dictum, supported as it has been in public by gentlemen in the same position as its author with a view of promoting certain legislation, is one strenuously to be combated, and it was my endeavour in the paper which I wrote in 1893 and published in 1894 in THE LANCET to prove that there were conditions connected with the mother to be taken into account in considering the causation of all puerperal fevers. At that time I was very much taken up with the consideration of the causation of puerperal thromboses. Since then a large experience of such cases and a large experience of women who were likely to suffer from eclampsia have led me to think more and more in the same direction as that in which Dr. Allbutt points, though without the same exact knowledge of the chemistry involved.

What is the basis upon which my thoughts rest? To take the subject of Dr. Allbutt's paper first, and a case which recently gave me grave anxiety before delivery. The history of the patient: Five deliveries, one child stillborn; a great many miscarriages; puerperal sepsis twice, once leading on to parametric abscess ; at the last delivery eclampsia. The patient was about thirty-five years of age, and at my first examination there was albumin in the urine. There was also general anasarca, well marked but not excessive. This history was sufficient in itself not only to arouse special interest, but special anxiety for the result. The patient was therefore placed upon a treatment of purgation accompanied by treatment by iron, and a diet in which milk was pushed. Two scruples of compound jalap powder and one of the acid tartrate of potassium were given in the first place without result. This was followed by an active purgative pill, which produced an evacuation. Subsequently the patient took, night and morning, a drachm of Epsom salts and fifteen drops of the liquid cascara extract with some ginger. The result was satisfactory, for two or three liquid actions of the bowels resulted every day. Besides this, a mixture containing the freshly precipitated oxide of iron was given thrice daily.
The labour came on at the end of the eighth month, and was terminated so far as the child was concerned before my arrival. The albumin in the urine at once diminished, and the patient made an excellent recovery. The treatment, which is a time-honoured one in this practice, is one which have carried out because it is based upon a working hypothesis. Pargatives which produce free watery evacuations have an action upon the blood. What that action is cannot be definitely stated, but Dr. Allbutt's paper indicates a possible, if not a probable, one, and the same remark applies to the action of iron salts.

Other conditions connected either directly or indirectly with pregnancy seem to me to support Dr. Allbutt. First, the odematous condition not infrequently met with in pregnant women, with no albumin in the urine and with no eclampsia. These women, although often so swollen that they cannot wear their rings, do not suffer in health; but surely this is a condition which we can connect with the more serious one. Secondly, there is the condition which the late Dr. Matthews Duncan described in his lecture upon Hydroperitoneum - the collection of fluid in the abdominal cavities of elderly women who have borne children without any sign or symptom of disease of organs. Here, again, is a condition difficult of explanation no doubt, but eminently one to be considered in connexion with the subject. Thirdly, there are the various conditions connectea with the vomiting of pregnancy. The late Dr. Matthews Duncan fully recognised the fact "that the condition of the blood is probably a chief part of the cause of the proneness of women to disease of the kidney and uræmia," the condition of the great glands being, in his opinion, "probably the cause of their proneness to further dangerous stages of parenchymatous degeneration, chiefly of the liver, but also of the kidneys and other organs." He looked upon the parenchymatous degeneration of the great glands as the first stage of the serious cases of vomiting in pregnancy, the degeneration present even in healthy pregnancies. It is not very difficult now to suggest the link in the causation of such troubles if Dr. Allbutt's theory is accepted. Dr. Duncan, with his great shrewdness and unrivalled experience, knew that the conditions connected with certain cases of the vomiting of pregnancy were not due to nerrous causation, but be did not know what is now known of chemical conditions connected with the blood. He pointed to the liver as being the organ most at fault, and in this he was doubtless correct ; the knowledge of his day showed that there were changes in the structure of that organ, but it did not show that there were chemical substances circulating in the blood of pregnant women which might account for the change in its structure as well as for that in other glands. His teaching on this subject is, however, only part of the debt which his pupils owe to him, and it is a matter of interest to read Dr. Allbutt's paper with that teaching present to the mind.

Now, to take the subject of thromboses. These have occurred in $\mathrm{my}$ practice at times varying between ten days and the fourth week after delivery, with no rise of temperature or any indication of sepsis before the date of the attack. It certainly appears unlikely that there is a connexion between these attacks and sepsis at the time of delivery, and an explanation based upon the theory propounded by Dr. Allbutt appears more reasonable and is certainly that to which I incline. The one predisposing factor at the time of delivery which has been brought forward as conducive to these cases is hæmorrbage, and in none of the three cases which have occurred in my practice has bæmorrhage been present of such a character as to call for remark. The late Dr. Wooldridge's paper on Auto-infection in the Causation of Cardiac Disease certainly leads me in the direction of belief that these thromboses are due to the presence in the blood of some chemical substance peculiar to the blood of puerperal women. It also lends support to Dr. Allbutt's paper.

Finally, what evidence will a study of puerperal fevers provide? Let us put aside once for all statistics, which can be of no possible utility in the consideration of causation. As every working obstetrician knows full well, it is impossible to forecast the progress of a patient during the lying.in period. The cases which have demanded great attention and caused anxiety during the actual labour are not those which, as a rule, give further anxiety, and I include the third stage of labour, which is the critical one so far as puerperal fevers are concerned. It is the critical one because, in the first place, of the difficulty of certainty as to complete removal of all decidual remnants, 